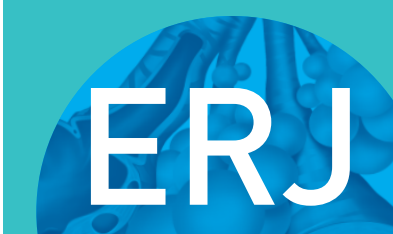

open research
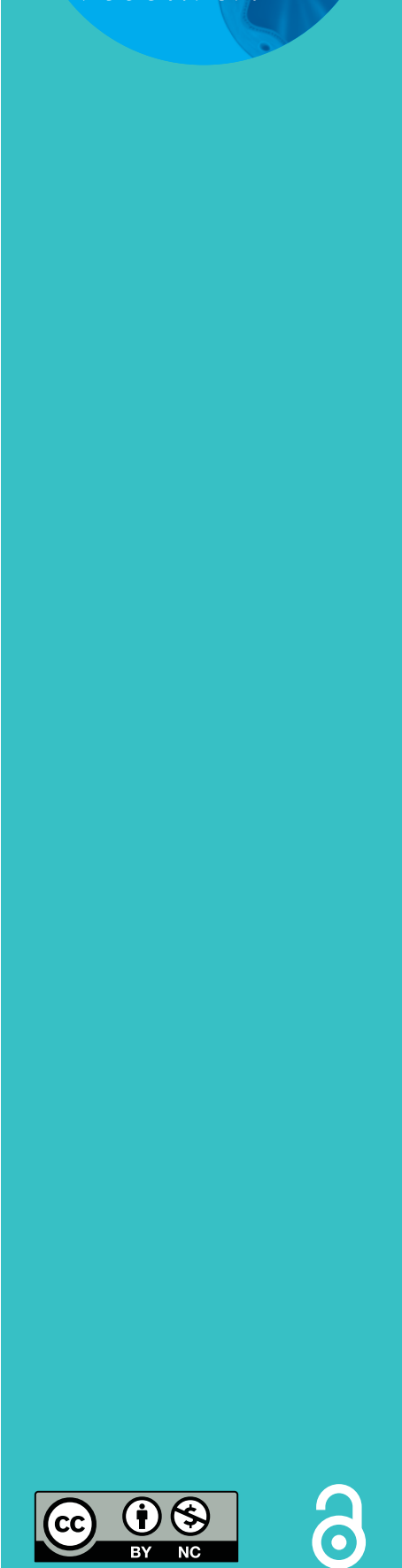

\section{An audit of COPD: diagnosis and management in general practice}

\author{
Ghassan Hamad, Alan Rigby and Alyn H. Morice
}

Affiliation: Hull York Medical School, Castle Hill Hospital, Hull, UK.

Correspondence: Ghassan Hamad, University of Hull, Respiratory Medicine Dept, Castle Hill Hospital, Hull, HU6 7RX, UK. E-mail: ghassanhamaddyahoo.co.uk

\section{ABSTRACT}

Introduction: COPD is a spectrum of disorders primarily caused by smoking and characterised by progressive, not fully reversible airflow obstruction with a forced expiratory volume in $1 \mathrm{~s}\left(\mathrm{FEV}_{1}\right) /$ forced vital capacity $(\mathrm{FVC})$ ratio $<0.7$.

Methods: From November 2016 to March 2017 we audited patients with COPD in five general practices in Hull and East Riding, UK. We looked at deviation from the locally agreed guidelines. We extracted data on severity, exacerbations, medication and eosinophil count.

Results: We assessed 1088 records. Median age was 70.9 years; 577 (53\%) were male. About two-thirds of patients on the COPD register have an $\mathrm{FEV}_{1} / \mathrm{FVC}$ ratio in the diagnostic range for $\mathrm{COPD}$, however, 388 $(36 \%)$ out of 1088 had a ratio of $\geqslant 0.7$. In the patients with a ratio of $\geqslant 0.7,259(67 \%)$ out of 388 had an $\mathrm{FEV}_{1}<80 \%$ of predicted. Patients with frequent exacerbations were more likely to be prescribed inhaled corticosteroid (ICS)-containing inhalers (incidence rate ratio of 2 ). $\mathrm{FEV}_{1} \%$ predicted was a poor indicator of exacerbation frequency; however, the presence of elevated blood eosinophil counts (EOS) on at least two occasions was highly predictive of exacerbations. When ICSs, FEV 1 , EOS were examined in combination, they were highly significant predictors for exacerbations.

Conclusion: $\mathrm{FEV}_{1}$ maybe a more accurate diagnostic parameter in primary care. Historical evidence of blood eosinophilia is a better predictor than $\mathrm{FEV}_{1}$. The combination of biomarkers may prove more accurate indicator of future exacerbation frequency, leading to targeted intervention.

@ERSpublications

FEV $_{1}<80 \%$ predicted provides a greater concordance with the primary care clinical diagnosis of

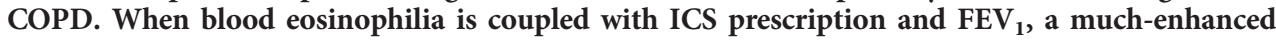
predictability of exacerbation is achieved. https://bit.ly/2PtYS83

Cite this article as: Hamad G, Rigby A, Morice AH. An audit of COPD: diagnosis and management in general practice. ERJ Open Res 2020; 6: 00330-2020 [https://doi.org/10.1183/23120541.003302020]. 


\section{Introduction}

COPD is a spectrum of disorders primarily caused by smoking and characterised by progressive, not fully reversible airflow obstruction [1]. It is a major public health concern with an estimated $2 \%$ of the United Kingdom population (1.2 million people) having diagnosed COPD [2]. With as many patients going undiagnosed, a more accurate figure would be at least twice this number [2]. The UK National Institute for Health and Care Excellence (NICE) recommends COPD to be suspected in patients aged over 35 years with a significant history of smoking and who have the appropriate chronic symptoms of breathlessness, cough and sputum. This is then confirmed with forced expiratory volume in $1 \mathrm{~s}\left(\mathrm{FEV}_{1}\right) /$ forced vital capacity (FVC) ratio of $<0.7$ [3].

Guidelines advocate the use of personalised approach to prescribing in stable COPD. The latest NICE guidelines [3] and our local guidelines in Hull and East Riding [4] advocate long-acting $\beta$ adrenoceptor agonist (LABA)/long-acting muscarinic antagonist (LAMA) combination inhalers as a first step if intermittent short acting bronchodilators are no longer adequate. In our guidelines, the next step is to add inhaled corticosteroids (ICSs) if blood eosinophilia is present. This guidance is based on a growing body of evidence to suggest that the treatable trait of ICS response is most effective in COPD patients with an element of eosinophilic airway inflammation (i.e. the asthma-COPD overlap) [5, 6]. There is also good evidence that blood eosinophil count, a readily accessible clinical aid in general practice, is a good surrogate marker for bronchial eosinophilia that could be used to guide ICS therapy [7, 8].

We undertook an audit of patients with a diagnosis of COPD from five local general practices. In this study we report on the relationship of demographics, disease severity and blood eosinophil counts with exacerbation rates. Here we report on the first cycle of the audit programme.

\section{Methods}

From November 2016 to March 2017 we audited patients with COPD in five general practices in Hull and East Riding, UK. We looked at deviation from the locally agreed guidelines. To deal with incomplete coding of data in the electronic records, data were completed by manual extraction. We extracted data on COPD severity, exacerbations, medication, eosinophil count and healthcare utilisation. We looked at the last available five full blood counts and considered raised eosinophil count to be present if at least one count out of the last five counts was over $0.3 \times 10^{9} \cdot \mathrm{L}^{-1}$. For this audit we defined exacerbations as two or more episodes of worsening symptoms requiring antibiotic and/or oral steroid or one or more hospital admission in the year preceding data collection.

According to the Health Research Authority online assessment [9] no ethics approval was needed as this was a service improvement activity.

\section{Statistical methods}

Continuously distributed data are summarised by the median (25th/75th centiles); categorical data are $\mathrm{n}(\%)$. The relationships between exacerbations, eosinophil counts and the use of inhalers were examined using Poisson regression. A requirement of Poisson regression is that the mean number of exacerbations should be equal to its variance. Where the variance is greater than the mean, negative binomial regression is preferred to Poisson. An arbitrary level of significance of $5 \%$ was assumed (two-tailed). The STATA computer package was used to analyse the data [10].

\section{Results}

In total, the electronic records of 1088 patients with a recorded diagnosis of COPD were accessed. Median age was 70.9 years (interquartile range (IQR) 63.3-79.5); 577 (53\%) were males. About 10\% (110 out of 1088 ) of the study population were recorded as never smoked, $58 \%$ (625 out of 1088) as ex-smokers and $32 \%$ (352 out of 1088) as current smokers. We gathered the latest entered values of $\mathrm{FEV}_{1} / \mathrm{FVC}$ on records. Overall, 1034 (95\%) patients had at least one spirometry on their medical records. The median $\mathrm{FEV}_{1} / \mathrm{FVC}$ ratio was 0.66 (IQR 0.56-0.75). About two-thirds of patients on the COPD register had an $\mathrm{FEV}_{1} / \mathrm{FVC}$ ratio in the diagnostic range for COPD; however, 388 out of $1088(36 \%)$ had a ratio of 0.7 or greater. In the patients with a ratio of 0.7 or greater, the median $\mathrm{FEV}_{1}$ was $69 \%$ and of these 259 out of $388(67 \%)$ had an $\mathrm{FEV}_{1}<80 \%$ of predicted. Thus, of the total population about one-third would not meet the guideline-specified $\mathrm{FEV}_{1} / \mathrm{FVC}$ criteria for airflow obstruction. However, of these with a guideline-specified normal ratio, two-thirds (one-quarter of the total population) had an $\mathrm{FEV}_{1}<80 \%$ predicted.

When patients were categorised according to the NICE classification, 38 out of 1088 (3\%) had very severe obstruction $\left(\mathrm{FEV}_{1}<30 \%\right.$ pred), 203 out of 1088 (19\%) had severe obstruction ( $\mathrm{FEV}_{1}$ 30-49\% pred), 535 out of 1088 (52\%) had moderate obstruction ( $\mathrm{FEV}_{1} 50-79 \%$ pred) and 258 out of 1088 (24\%) had mild obstruction $\left(\mathrm{FEV}_{1} \geqslant 80 \%\right.$ pred). Of the latter group, 146 out of $258(57 \%)$ had FEV1/FVC ratio $<0.7$. Thus, 

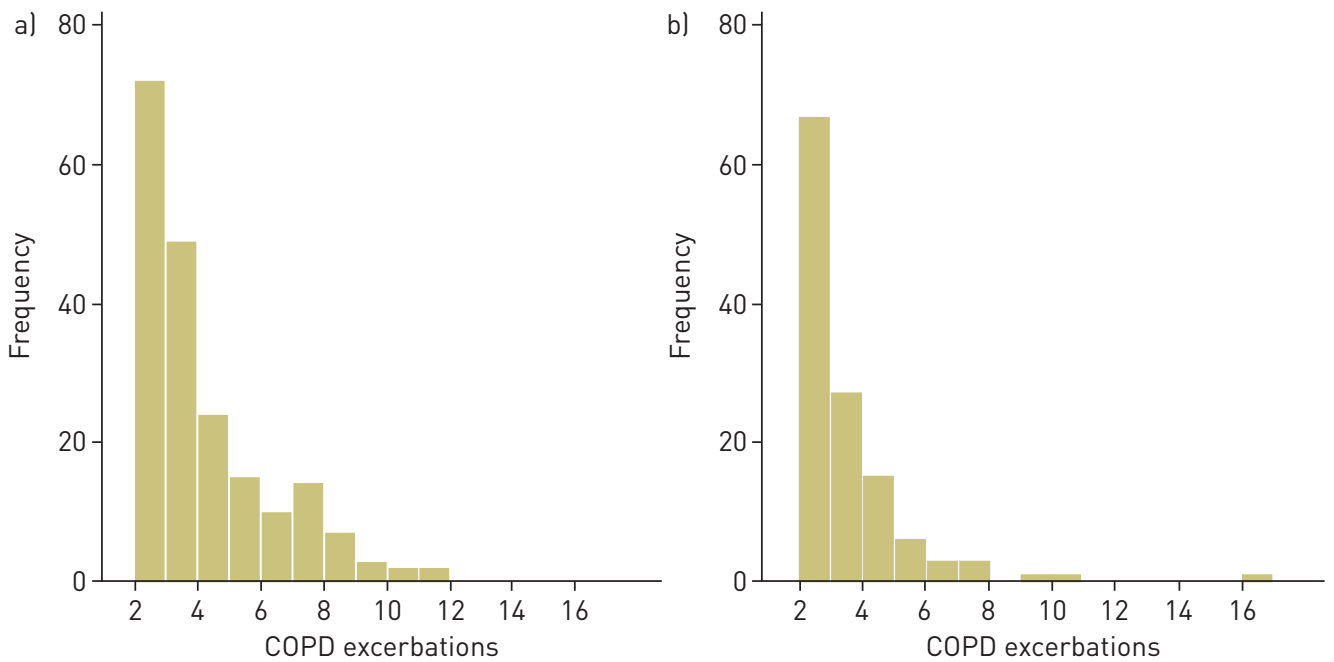

FIGURE 1 Number of exacerbations by inhaled corticosteroid (ICS) usage. a) ICS inhaler (mean number of exacerbations 1.38) and b) no ICS inhaler (mean number of exacerbnations 0.69).

approximately one-tenth of all patients had a normal $\mathrm{FEV}_{1} / \mathrm{FVC}$ ratio with an $\mathrm{FEV}_{1}>80 \%$ pred, suggesting lung physiology within normal limits.

Most patients (74\%; 804 out of 1088) had at least one full blood count on their primary care records. Of those, 63\% (504 out of 804 ) had at least one eosinophil count above $0.3 \times 10^{9} \cdot \mathrm{L}^{-1}$. Recorded asthma co-diagnosis was observed in 319 out of 1088 (29\%) patients. In patients with an asthma co-diagnosis, only 83 out of 319 (26\%) had a raised eosinophil count.

Almost half of the patients (548 out of 1088) were on regimes that do not contain ICS. About 14\% (148 out of 1088) of patients had a history of two or more COPD exacerbations or at least one hospital admission in the previous year. Patients with frequent exacerbations were more likely to be prescribed an ICS-containing inhaler (overall incidence rate ratio (IRR) of 2; i.e. twice as likely) (figure 1, table 1). The IRR of patients on dual inhaled therapy containing ICSs (i.e. LABA/ICS or LAMA/ICS) was 1.38 (95\% CI $1.15-1.65 ; \mathrm{p}<0.0001$ ), whereas the IRR of patients on triple inhaled therapy (LABA/LAMA/ICS) was 2.42 (95\% CI 2.17-2.76); $\mathrm{p}<0.0001)$. Furthermore, 170 patients had frequent exacerbation and raised eosinophil counts. Patients with frequent exacerbations and raised eosinophil counts had an IRR of 1.4-times greater than ICS prescribing. Predicted $\mathrm{FEV}_{1} \%$ was a poor indicator of exacerbation frequency. Excluding patients with an $\mathrm{FEV}_{1} / \mathrm{FVC}$ ratio of $<0.70$ ( 0.36 of patients) did not influence the observed exacerbation rate (table 1).

There was no significant increase in exacerbation rate in smokers compared to never smokers (IRR 1.05, 95\% CI $0.78-1.47, \mathrm{p}=0.72$ ). There was only a weak correlation seen between $\mathrm{FEV}_{1} \%$ predicted and the number of exacerbations observed in the preceding year (figure 2). The distribution of exacerbations was poorly correlated with the prescription of ICSs (figure 1). In contrast, peripheral blood eosinophilia $\left(\geqslant 0.3 \times 10^{9} \cdot \mathrm{L}^{-1}\right)$ was associated with an increase in exacerbation frequency (figures 3 and 4$)$. However, with a single elevated blood eosinophil count, the association was weak and nonsignificant. When blood eosinophil counts were raised on at least two occasions, this was highly predictive of exacerbations. The incidence rate for at least one exacerbation was 1.62-times greater than the incidence of those without any eosinophilia. Patients with lower $\mathrm{FEV}_{1} \%$ predicted are more likely to have exacerbations (table 1).

TABLE 1 COPD exacerbations: relationship to raised eosinophil count (EOS), inhaled corticosteroid (ICS) treatment and forced expiratory volume in $1 \mathrm{~s}\left(\mathrm{FEV}_{1}\right)$

\begin{tabular}{|c|c|c|c|c|c|}
\hline Variable & Univariable & $(95 \% \mathrm{CI})$ & p-value & $\begin{array}{c}\text { Excluding } \mathrm{FEV}_{1} / \mathrm{FVC} \text { ratio }>0.70 \\
\text { univariable }(95 \% \mathrm{CI})\end{array}$ & p-value \\
\hline EOS at least 1 value $\geqslant 0.3 \times 10^{9} \cdot \mathrm{L}^{-1}$ & 1.62 & $(1.23-2.15)$ & 0.0006 & $1.75(1.50-2.05)$ & $<0.0001$ \\
\hline EOS $\geqslant 2$ readings & 1.63 & $(1.11-2.40)$ & 0.013 & $1.84(1.48-2.78)$ & $<0.0001$ \\
\hline ICS inhaler & 2 & $(1.51-2.63)$ & $<0.0001$ & $2.13(1.81-2.51)$ & $<0.0001$ \\
\hline $\mathrm{FEV}_{1} \%$ predicted & 0.98 & $(0.97-0.99)$ & $<0.0001$ & $0.98(0.98-0.99)$ & $<0.0001$ \\
\hline
\end{tabular}


FIGURE 2 Scatter plot of forced expiratory volume in $1 \mathrm{~s}\left(\mathrm{FEV}_{1}\right)$ versus number of exacerbations in patients with $>1$ exacerbation. National Institute for Health and Care Excellence severity cut-off points are indicated by red vertical lines. Correlation: -0.16 .

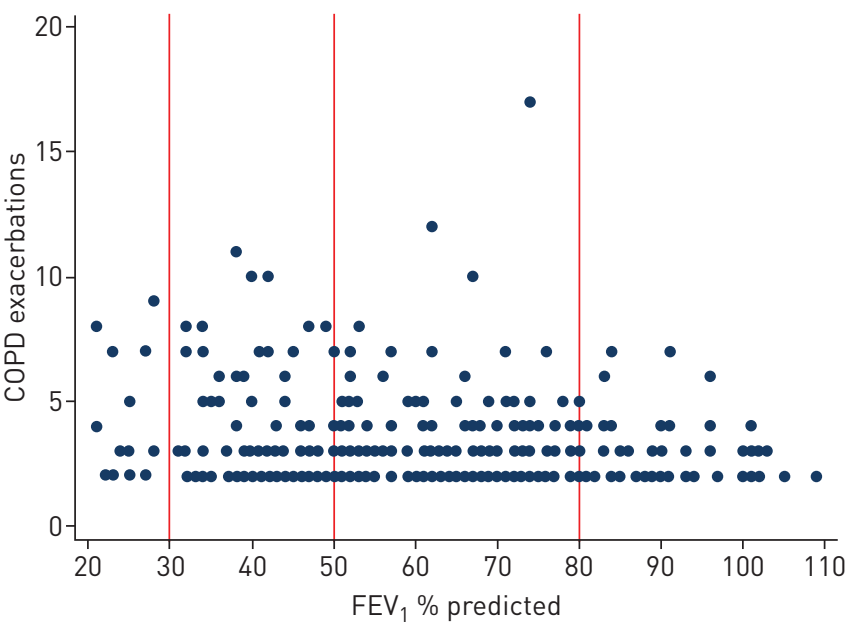

When all three predictors of exacerbation rate (ICS, $\mathrm{FEV}_{1}$, raised eosinophil count) were examined in combination, they were highly significant predictors for exacerbations, doubling the ability to predict an exacerbation (figure 5).

\section{Discussion}

The primary objective of this audit was to investigate the diagnostic accuracy and the current prescribing in primary care compared to that recommended by local and international guidelines.

The 2018 NICE guidance NG115 [3] recommends the use of spirometry to confirm the diagnosis of COPD. GOLD guidelines also define airway obstruction using the fixed ratio of $\mathrm{FEV}_{1} / \mathrm{FVC}$ of 0.70 or less [11]. In contrast, the severity of the airflow obstruction in COPD is classified by the degree of reduction in $\mathrm{FEV}_{1} \%[3,11]$.

General practitioners in the UK are contracted to offer spirometry for potential COPD sufferers but, at the time of undertaking this audit, only $\mathrm{FEV}_{1}$ is reimbursed when performed annually. From April 2019 measurements of $\mathrm{FEV}_{1}$ are no longer required on an annual basis to guide care [12].

It has long been acknowledged that operator, patient and equipment factors are the most common reasons for inconsistent spirometry readings $[13,14]$. In primary care, the spirometry is usually performed by
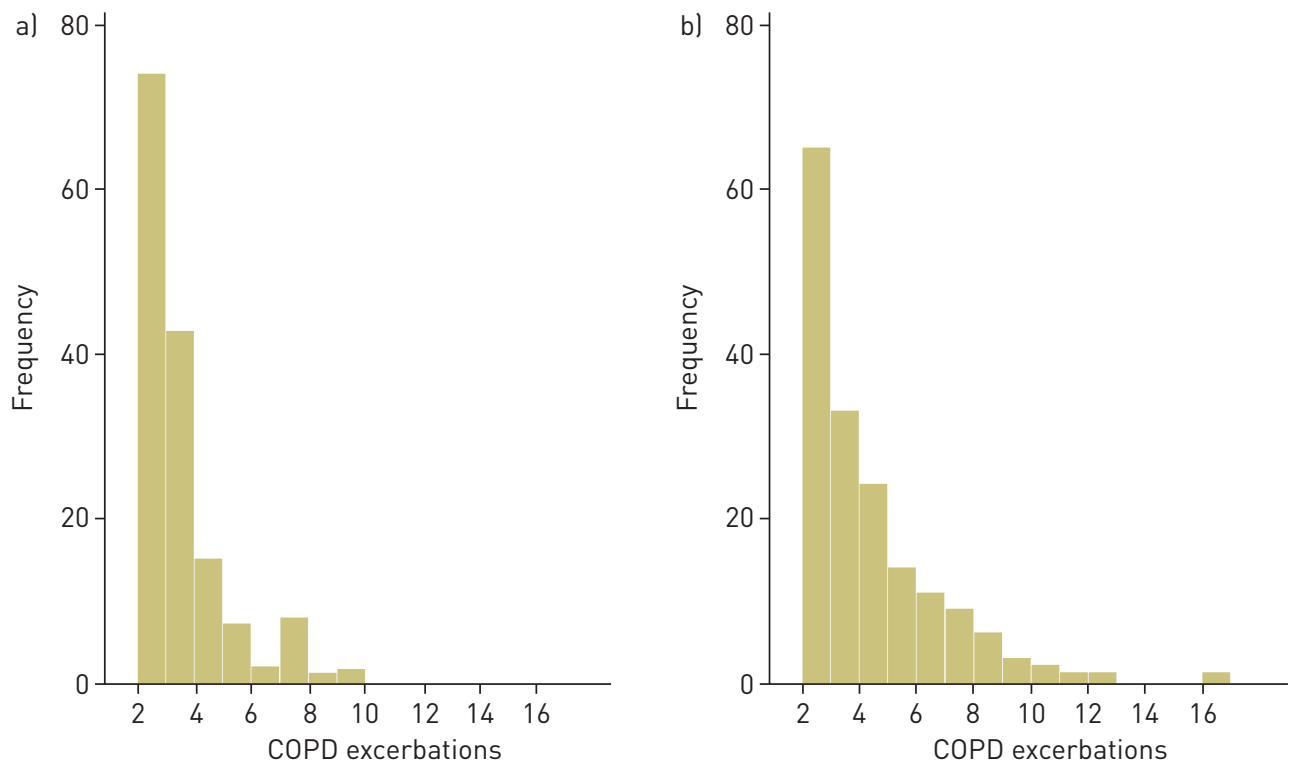

FIGURE 3 Number of exacerbations by presence or absence of elevated blood eosinophil count (EOS). a) No EOS (mean number of exacerbations 0.80 ) and b) at least one positive EOS reading (mean number of exacerbations 1.30). 

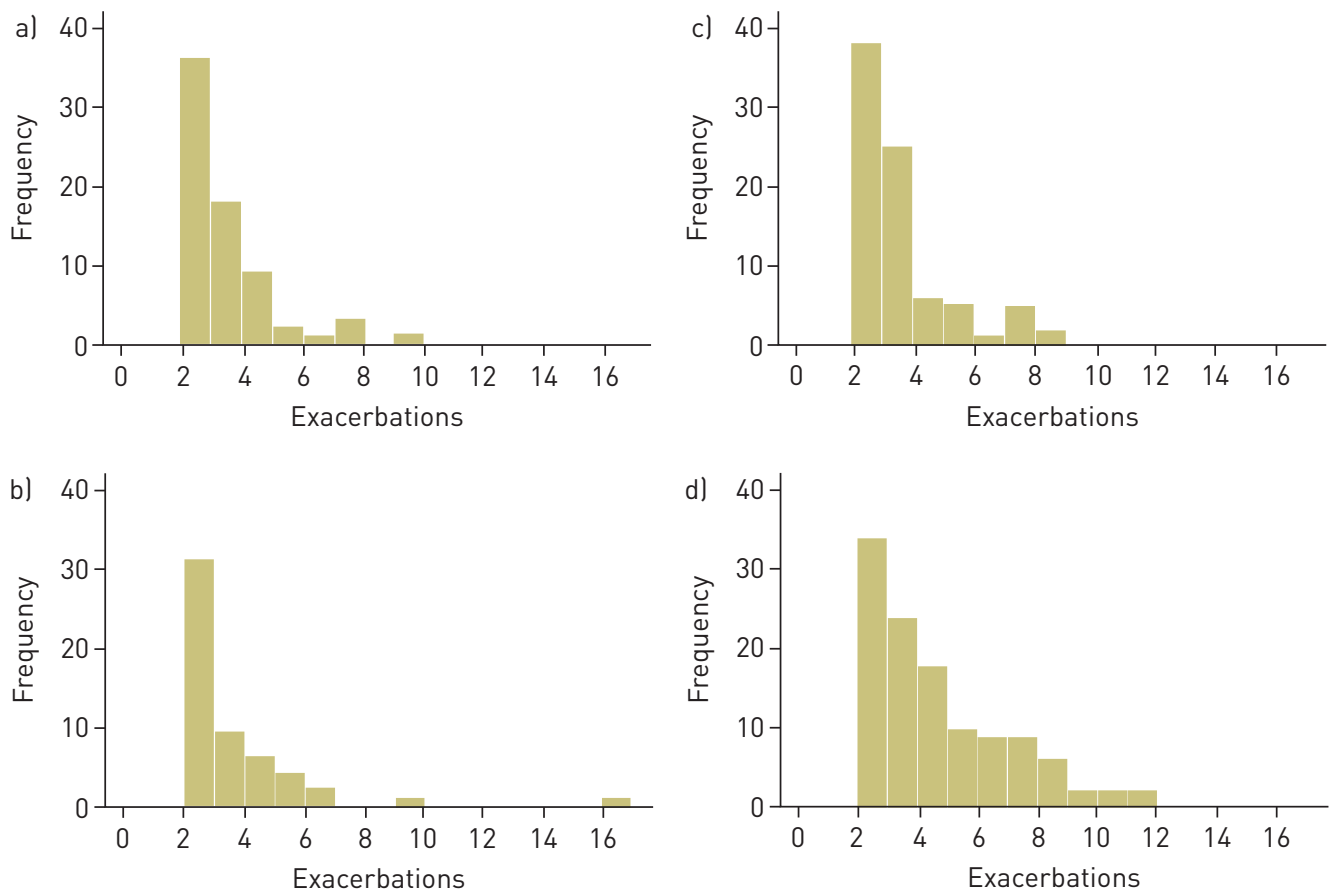

FIGURE 4 Number of exacerbations by both inhaled corticosteroid (ICS) usage and elevated blood eosinophil count (EOS). a) No ICS use, no EOS (mean 0.65, 25th-75th percentiles 0-0), b) no ICS use, EOS (mean 0.75, 25th-75th percentiles 0-2), c) ICS use, no EOS (mean 0.97, 25th-75th percentiles 0-1), and d) ICS use, EOS (mean 1.76 , 25th-75th percentiles $0-3$ ).

trained nurses, at the point of COPD diagnosis and, prior to April 2019, annually thereafter to detect the rate of decline in lung function. The NICE guideline recommends that all healthcare professionals managing patients with COPD should be competent in the interpretation of the results of spirometry and should have undergone appropriate training and keep their skills up to date.

The results of our audit suggest that if the $\mathrm{FEV}_{1} \%$ predicted was used as a diagnostic criterion, then more patients with COPD would meet the spirometric criteria for the clinical diagnosis for COPD in primary care. We understand the rationale for using the $\mathrm{FEV}_{1} / \mathrm{FVC}$ ratio in secondary care as other rarer conditions such as interstitial lung disease may cause a reduction in $\mathrm{FEV}_{1}$. However, these conditions are usually characterised by abnormalities on the chest radiograph or on examination and can be excluded during the diagnostic workup. We suggest that in primary care a more practical diagnostic criteria for COPD would be the use of $\mathrm{FEV}_{1} \%$ predicted in the presence of a normal examination and a chest radiograph.

When we consider exacerbation frequency, one of the most predictive biomarkers was shown to be historical blood eosinophil counts. Those patients with elevated counts $>0.3 \times 10^{9} \cdot \mathrm{L}^{-1}$ had greater than $50 \%$ increase in exacerbation frequency. Further analysis demonstrated that almost all of this increase was

FIGURE 5 Predicted number of COPD exacerbations in the year preceding data collection from inhaled corticosteroid (ICS) inhaler use, elevated blood eosinophil count (EOS) and forced expiratory volume in $1 \mathrm{~s}\left(\mathrm{FEV}_{1}\right)$. Pairwise comparisons indicated ICS+EOS was significantly different from the other three groups, but no other significant differences were seen.

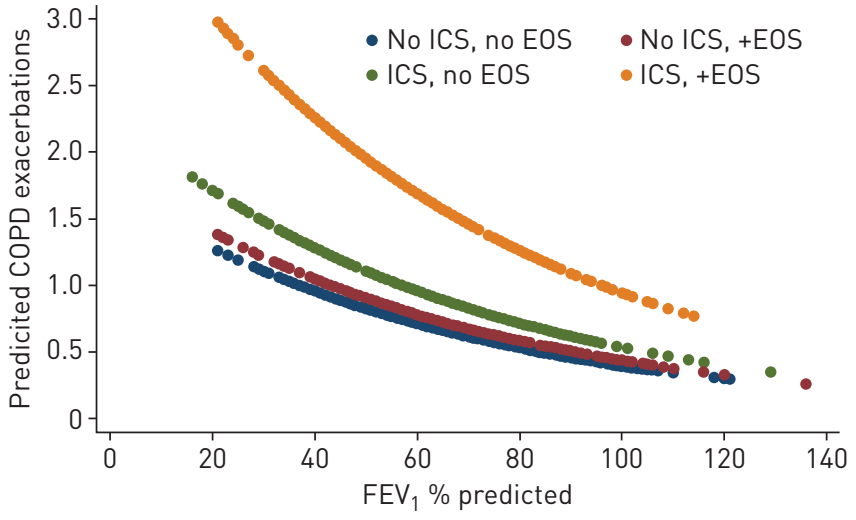


found in those patients that had a persistent or intermittent eosinophilia. This was similar to that found in our previous study which showed that greater or equal than two elevated eosinophil counts $>0.3 \times 10^{9} \cdot \mathrm{L}^{-1}$ was highly predictive of exacerbation frequency [15]. ICS use was associated with increased exacerbation frequency, especially those on triple inhaled therapy containing ICSs, although whether this was in response to guideline recommendation for ICS use in frequent exacerbators is unclear. There was however no indication that frequent exacerbators had a greater propensity for inhaled ICS prescription (figure 1). When we examined \% predicted $\mathrm{FEV}_{1}$ on exacerbation frequency there was only a poor predictive value with an IRR of 0.98 .

We next examined whether combining the various markers of exacerbation frequency could enhance the prediction of exacerbation frequency over individual measures. Figure 5 illustrates that when blood eosinophil count is coupled with ICS prescription and $\mathrm{FEV}_{1}$ a much-enhanced predictability of exacerbation was achieved. In future it may be possible to produce a simple tool to guide the clinician to identify those at greater risk of exacerbation thus aid personalisation of their treatment.

\section{Conclusions}

In summary, we have demonstrated that in a group of over 1000 patients with a clinical diagnosis of $\mathrm{COPD}$ in primary care, $\mathrm{FEV}_{1} \%$ predicted $<80 \%$ provides a greater concordance with the primary care clinical diagnosis. There are many problems with the use of spirometry in primary care, including staff training issues, time pressures and, more recently, the lack of financial incentive to undertake all the components of the spirometry beyond the point of initial inclusion in the COPD register. FVC is a more difficult manoeuvre to perform correctly in a primary care setting. We would suggest that $\mathrm{FEV}_{1} \%$ predicted may represent a more practical tool for diagnosing airway obstruction, having excluded other rarer diagnoses. To predict the major morbidity in COPD, the exacerbation, we have demonstrated that historical evidence of blood eosinophilia is a better predictor than $\mathrm{FEV}_{1}$. The combination of biomarkers may prove more accurate indicator of future exacerbation frequency leading to targeted interventions. In this audit the blood eosinophil count appears to be under-utilised as a guide to personalised therapy. Future development of a guideline-led combination tool may aid the reduction of this costly morbidity.

Conflict of interest: G. Hamad reports grants from AstraZeneca during the conduct of the study. A. Rigby has nothing to disclose. A.H. Morice reports grants from AstraZeneca during the conduct of the study.

\section{References}

1 Sutherland ER, Cherniack RM. Management of chronic obstructive pulmonary disease. N Engl J Med 2004; 350: 2689-2697.

2 Snell N, Strachan D, Hubbard R, et al. S32 Epidemiology of chronic obstructive pulmonary disease (COPD) in the UK: findings from the British Lung Foundation's 'Respiratory Health of the Nation' project. Thorax 2016; 71: A20.

3 National Institute for Health and Care Excellence. Chronic obstructive pulmonary disease in over 16s: diagnosis and management. NICE guideline. Date last accessed: 12 August 2020. Date last updated: 5 December 2018. www. nice.org.uk/guidance/ng115.

$4 \quad$ Hull and East Riding Prescribing Committee. Prescribing guidelines, COPD treatment pathway, 2018. www.hey. nhs.uk/wp/wp-content/uploads/2016/03/copdTreatmentPathway.pdf Date last updated: March 2018; date last accessed: September 2019 .

5 Hinds DR, DiSantostefano RL, Le HV, et al. Identification of responders to inhaled corticosteroids in a chronic obstructive pulmonary disease population using cluster analysis. BMJ Open 2016; 6: e010099.

6 Vogelmeier CF, Criner GJ, Martinez FJ, et al. Global strategy for the diagnosis, management, and prevention of chronic obstructive lung disease 2017 report: GOLD executive summary. Eur Respir J 2017; 49: 1700214.

7 Pavord ID, Lettis S, Locantore N, et al. Blood eosinophils and inhaled corticosteroid/long-acting $\beta$-2 agonist efficacy in COPD. Thorax 2016; 71: 118-125.

8 Cheng SL, Lin CH. Effectiveness using higher inhaled corticosteroid dosage in patients with COPD by different blood eosinophilic counts. Int J Chron Obstruct Pulmon Dis 2016; 11: 2341-2348.

9 Health Research Authority. Decision tool. www.hra-decisiontools.org.uk/research/ Date last accessed: 20 October 2016; date last updated: 20 October 2016.

10 StataCorp. Stata Statistical Software: Release 16. College Station, TX, StataCorp LLC, 2019.

11 Global Initiative for Chronic Obstructive Lung Disease. Global strategy for the diagnosis, management and prevention of chronic obstructive pulmonary disease (2018 report). https://goldcopd.org

12 Primary Care Strategy and NHS Contracts Group. 2019/20 General Medical Services (GMS) contract Quality and Outcomes Framework (QOF) Guidance for GMS contract 2019/20 in England April 2019. www.england.nhs.uk/ wp-content/uploads/2019/05/gms-contract-qof-guidance-april-2019.pdf Date last accessed: September 2019; date last updated: 30 April 2019.

13 Wat D, Sibley S. Accuracy of COPD diagnosis in primary care in Knowsley, UK. Eur Respir J 2017; 50: PA924.

14 Hegewald M, Gallo H, Linares O, et al. Accuracy of spirometers used in primary care. Eur Respir J 2015; 46: PA1577.

15 Hamad GA, Cheung W, Crooks MG, et al. Eosinophils in COPD: how many swallows make a summer? Eur Respir J 2018; 51: 1702177. 